\title{
A Syndromic Surveillance Service Supporting Environmental Public Health Incidents
}

\author{
Alex Elliot, Helen Hughes*, Roger Morbey, Paul Loveridge, Sally Harcourt, Sue Smith, \\ Zharain Bawa, Leandro Carrilho and Gillian Smith
}

Public Health England, Birmingham, United Kingdom

\section{Objective}

To deliver a national syndromic surveillance service, linking analytical and statistical methods with public health action to provide surveillance support for national public health programmes monitoring the spread of infectious diseases and the health impact of environmental incidents in England.

\section{Introduction}

Public Health England has developed a suite of syndromic surveillance systems, collecting data from a number of health care sources, and linking to public health action to try and improve the public health benefit of the surveillance. ${ }^{1}$ We aim to describe this national syndromic service, highlighting the flexibility of the systems in responding to a range of environmental incidents.

\section{Methods}

Syndromic surveillance data from general practitioners ('in hours' and 'out of hours'), sentinel ED attendances and telehealth calls were received on a daily basis using automated and secure data transfer processes. Data were analysed using a number of statistical and analytical processes generating statistically significant spikes in data, or increases compared to baselines.

\section{Results}

Syndromic surveillance reporting

Routine weekly surveillance bulletins are produced from each syndromic system: these reports present the current indicator trends and encapsulate the main summary findings from the weeks surveillance, providing a number of key messages for health professionals. ${ }^{1}$

\section{Heatwave surveillance}

Syndromic surveillance plays an important part in assessing the health impact of heatwaves and plays an integral role in the Heatwave Plan for England. During summer 2013 England experienced a heatwave: significant increases in heat/sunstroke were observed during 7-10 July 2013 (figure). ${ }^{2}$ In addition to the direct impact of heat, syndromic surveillance systems detected a spike in ED asthma attendances during a series of thunderstorms that followed the heatwave. ${ }^{3}$

Cold weather surveillance

A cold weather plan for England was launched in 2011 with the aim of reducing preventable mortality and morbidity due to severe cold weather. Syndromic surveillance plays a part in this plan: emergency department cold weather indicators have been developed and are used each winter to assess the impact of cold weather on public health. ${ }^{4}$

\section{Air pollution}

During March/April 2014 an air pollution event affected large parts of the UK; syndromic surveillance was used to assess the public health impact. Indicators for the presentation of asthma, difficulty breathing and wheeze all showed marked and significant increases during the air pollution episode, illustrating the impact of the episode.

Flooding

During early 2014 there was severe flooding across several areas of England. Syndromic surveillance systems were used, monitoring indicators of gastroenteritis, diarrhoea and vomiting. Daily data were compared between affected and unaffected areas to determine whether there had been an impact on health. There were no gross changes in the incidence of syndromic indicators in flooded areas, thus providing reassurance to national and local response teams.

\section{Conclusions}

Historically, syndromic surveillance systems have been primarily targeted towards supporting national infectious disease surveillance programmes e.g. influenza. However, the flexibility of the syndromic surveillance systems, and in particular the clinical indicators, allow these surveillance systems to monitor the health impact of environmental incidents.

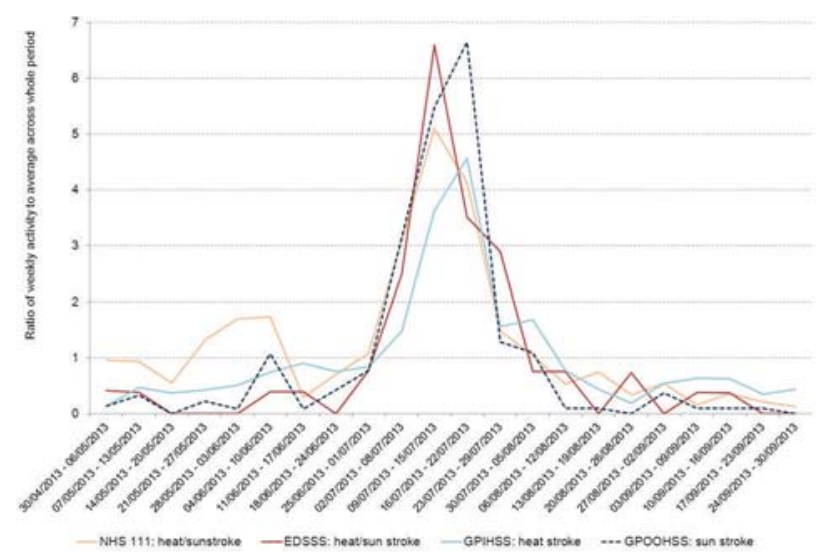

Syndromic surveillance 'heatwave' indicators during summer 2013

\section{Keywords}

syndromic surveillance; heatwave; flooding; air pollution; thunderstorm asthma

\section{References}

1. Public Health England. Real-time Syndromic Surveillance. 2014. https://www.gov.uk/government/collections/syndromic-surveillancesystems-and-analyses (accessed 29 Aug 2014).

2. Elliot AJ, Bone A, Morbey R, et al. Using real-time syndromic surveillance to assess the health impact of the 2013 heatwave in England. Environ. Res. 2014:doi 10.1016/j.envres.2014.08.031.

3. Elliot AJ, Hughes HE, Hughes TC, et al. The impact of thunderstorm asthma on emergency department attendances across London during July 2013. Emerg Med J 2014;31:675-8.

4. Hughes HE, Morbey R, Hughes TC, et al. Using an emergency department syndromic surveillance system to investigate the impact of extreme cold weather events. Public Health 2014;128:628-35.

\section{*Helen Hughes}

E-mail: helen.hughes@phe.gov.uk 